Katarzyna Woźniak

(Poznań University of Economics and Business, Poznań)

ORCID: 0000-0002-9077-328X

katarzyna.wozniak@ue.poznan.pl

\title{
MIGRATION POLICY IN THE AREA OF BORDER CONTROL AND MIGRATION OF THE POPULATION IN OECD COUNTRIES -THEORETICAL AND PRACTICAL ASPECTS
}

\begin{abstract}
The aim of the study is to present the phenomenon of population migration and migration policy as part of the state's economic policy based on the example of OECD (Organization for Economic Cooperation and Development) countries, with particular emphasis on the area of migration policy, which is border control and related illegal migration. The temporal scope of the empirical analysis covers the period 1990-2016. The article consists of four main parts. The discussion began with a presentation of the balance of migration, the scale and dynamics of population immigration in OECD countries. Furthermore, the significance, areas and process of shaping migration policy as a part of the economic policy of the country are presented. Then, it focused on the migration policy in the area of border control in OECD countries. The discussion was crowned with the conclusions that followed.
\end{abstract}

Keywords: OECD countries, migration, illegal migration, economic policy, migration policy doi: 10.2478/sho-2020-0010

\section{INTRODUCTION}

Migration flows of the population are an indispensable part of human history. The migration of people took on a special significance with the expansion of Europe in the 16th century. The mass flow of people from Europe to North America, which lasted from the mid-19th century to the First World War is considered to be the culmination of the development of population migration (also referred to in the literature as the "age of mass migration"). In turn, the migration flows initiated after 1945 (espe- 
cially escalating since the late 1980s) cover all regions of the world with international migration (as a result of political and cultural changes) at the centre of the globalization process [Castles S., Miller M.J. 2011: 19]. Decolonization was also an important factor that stimulated the flow of international migration. The probability of migration is particularly high in the case of countries that share a colonial past (i.e. colonial powers and their colonies) ${ }^{1}$ [Massey S.D. et al. 1993: 437-8].

The importance of the issue is primarily due to the fact that the adopted rules within the framework of migration policy have an impact on the distribution of costs and benefits resulting from migration for states, societies and individuals, as well as on the shape of the economic policy adopted by individual states. Management of population migrations is also a key element of the activities of many international organizations, including the Organization for Economic Cooperation and Development, the European Union, the United Nations and the International Organization for Migration. However, as indicated in the reports prepared by these institutions [Society at Glance... 2016: 7-8; Human Development Report... 2015: 163], population movements are constantly creating new challenges, both at the local and global levels.

The aim of the article is to present the phenomenon of population migration and migration policy as part of the state's economic policy in case of OECD countries. Special attention was paid to the area of migration policy, which is border control and related illegal migration. The article adopts two research theses. The first one indicates that migration policy is an important part of the state's economic policy, influencing other areas of socio-economic life. The second thesis assumes, in turn, that OECD countries are significantly different due to the scale and dynamics of population immigration, as well as in the enforcement of border control regulations.

An empirical analysis was carried out for the years 1990-2016. The choice of the beginning of the research period was based on two basic premises. First, the beginning of the 1990s was associated with many rapid changes in the social and economic order of the world, such as the collapse of the Soviet Union and Eastern European socialist countries. These events triggered destabilization in Central Europe, and removed many of the barriers that had previously been used to control the spatial mobility

${ }^{1}$ The impact of colonial ties on population immigration has also been confirmed in studies by R. de Arce and R. Mahia, [2008: 44-6] and A.M. Mayda [2010: 1263-5]. 
of the population. The changes in migration flows between the countries of Europe, North America, South America and Australia were also significant. In the mid-1980s, the countries of Southern Europe (which are reservoirs of labour for Western Europe, Australia, North America and South America) experienced a breakthrough in migration. Economic growth combined with a large decline in the number of births had led to labour shortages in these countries - turning them mostly into immigration countries (recording increasing inflows of labour, especially from Asia and Africa) [Castles S., Miller M.J. 2011: 141-2]. In turn, the year 2016 was the limit of the availability of statistical data.

The spatial scope of the empirical analysis covers 36 countries belonging to the Organisation for Economic Cooperation and Development $(\mathrm{OECD})^{2}$. The choice of countries to be analysed is based on two main considerations. First, it should be emphasized that some of the Organization for Economic Co-operation and Development (OECD) countries have been among the leading recipients of migration flows in the last century, which has resulted in significant economic, social and demographic changes in these countries. In the face of ageing populations, human migration has also become an important source of population growth [Grau Grau A.J., Ramírez López F. 2017: 2]. In addition, the historical and economic diversity of individual countries has provided a helpful empirical analysis of the heterogeneity of the countries studied.

The article consists of four main parts. The first part of the study presents the development of net migration, scale and dynamics of population immigration in OECD countries. The next part of the article presents the significance, areas, as well as the process of shaping the migration policy as part of the economic policy of the country. The third part of the article presents an analysis of the migration policy in the area of border control in the countries under study. The last part presents the main conclusions of the conducted analyses.

2 The Organisation for Economic Cooperation and Development (OECD), which was established in 1960 by 20 countries, includes, at present, the following countries: Australia, Austria, Belgium, Chile, Czech Republic, Denmark, Estonia, Finland, France, Germany, Greece, Hungary, Ireland, Iceland, Israel, Italy, Japan, South Korea, Latvia, Lithuania, Luxembourg, Mexico, Netherlands, Norway, New Zealand, Poland, Portugal, Slovakia, Slovenia, Spain, Sweden, Switzerland, Turkey, USA, United Kingdom, and Colombia. Because Colombia had just joined the OECD on 28 April 2020 - it was not included in this analysis [List of OECD... 2018; Colombia, 2020]. 


\section{IMMIGRATION IN THE OECD COUNTRIES BETWEEN 1990 AND 2016}

Despite the many diverse sources of statistical data on population migration, the use of the data obtained from them in other studies is associated with many limitations that are particularly related to the availability of statistical data, as well as to various definitions adopted by individual countries and organizations, which collect these data [Castles S., Miller M.J. 2011: 15].

The United Nations Statistics Division (UNSD) is a body authorized to collect official statistics on population migration through a data collection system. The Statistical Office of the European Union (Eurostat) has a similar task for Member States. Some countries also provide data on population migration to other international organizations, such as the Organization for Economic Cooperation and Development (IOM's Global Migration Data Analysis Centre [International migrant...2020] ${ }^{3}$. Taking into account the subject matter of this article, statistical data on international population immigration in countries belonging to the Organization for Economic Cooperation and Development are particularly important. Data on migration flows, especially including immigration in OECD countries can be found, among others, in databases of the United Nations and the Organization for Economic Cooperation and Development.

The United Nations provides statistical data on the total number of international migrants who have entered a country in a given calendar year through citizenship and country of birth. In countries where the number of immigrants is based on the number of residence permits issued, data is available only about foreigners. However, in most countries the data includes both foreigners and citizens. For countries that define international migrants based on citizenship, a distinction between foreigners and citizens is available by definition. In most countries where international migrants were defined based on country of residence, no distinction between foreigners and citizens is made [UN DESA 2015]. However, for OECD countries, this data was only available until 2013. Adopting

\footnotetext{
3 There are also many studies that contain data on bilateral migration flows between countries (for example, the DEMIG database (International Migration Institute, 2020), which collected data on bilateral population flows for 236 countries between 1946 and 2011, although it should be noted that the time range of data availability varies between countries.
} 
these data for analysis would significantly reduce the time ranges of the study, especially preventing the analysis from taking into account the migration dynamics in recent years. The limited comparability of the data is another difficulty in using it. For example, data on immigration to the U.S. are based solely on the criterion of "place of birth" and concern only foreigners. However, in the case of Australia, available data are based on the criterion of "country of residence", covering both citizens and foreigners.

The Organization for Economic Cooperation and Development, on the other hand, publishes statistical data on the inflow of foreign population estimated based on the number of permits issued in a given period (for permanent and temporary residence), as well as the inflow of immigrants for permanent residency. Although data on the inflow of foreign population have been available since 1975, they were already available for individual OECD countries in the 1970s. In the 1990s, however, the availability of these data increased significantly, and for most OECD countries the data was available throughout the research period adopted in the paper. Individual variables are differentiated according to two criteria: gender and country of birth [Foreign population 2018]. An additional advantage of these data is the inclusion of both permanent and temporary immigration.

Based on the analysis of statistical data published by international organizations, such as the International Organization for Migration, the United Nations, the Organization for Economic Cooperation and Development as well as the European Union, in order to present the diversity of immigration in the OECD countries between 1990 and 2016. The Organization for Economic Cooperation and Development definition was adopted, according to which population immigration was estimated on the basis of the number of permits issued in a given period (for permanent and temporary residence) [International Migration Database... 2018].

It should be added, however, that the Organisation for Economic Cooperation and Development also provides data on the inflow of immigrants for permanent residence. Data on inflow of immigrants for permanent residence include recorded movements of persons who are considered to be settling in a particular country (from the point of view of the host country). These data is the result of a standardization process that allows for cross-country comparative analysis [Permanent immigrant... 2018]. However, these data are available for a relatively short span of time. For most OECD countries the available data cover the period 2004-2016. 
The literature also points to many doubts and limitations in basing empirical analyses only on permanent migration. As noted by A. Górny and P. Kaczmarczyk [2003: 7-8, after: Chapman M., Prothero M. 1985; Hugo G. 1982; Kaczmarczyk P. 2002], the use of the category of settlement migration leads to the study of the abstract category, because people are constantly in motion and settlement is one of many forms of migration. Thus, omission of time migration in the analyses can lead to false conclusions and a false picture of reality. Moreover, a particular person may have more than one residence or, in extreme cases, may not have at all [Górny A., Kaczmarczyk P. 2003: 7-8, after: Chapman M., Prothero M. 1985; Hugo G. 1982; Kaczmarczyk P. 2002] $]^{4}$. Although the use of OECD data on population immigration estimated, based on the number of permits issued during a given period (permanent and temporary residence), has some limitations - it allows to include most of the countries belonging to the Organization in the quantitative analysis, as well as to take into account the broadest possible temporal range of the conducted analyses.

The coefficients used for comparative analyses of the intensity of demographic phenomena are the measure of the intensity of demographic phenomena in a unit of time ${ }^{5}$ [Okólski M., Fihel A. 2012: 16, 24-7, 48]. In order to present the development of the volume and dynamics of population immigration in the OECD countries, the changes in net migration will be discussed first ${ }^{6}$.

Figure 1 presents a reference of net migration relative to the population in a given country. In 1990, the size of the net migration in OECD countries ranged from -6.5 in Ireland to 8.1 number of people (per 1000 people) in Australia. Consequently, in 2015, the lowest value of net migration was re-

${ }^{4}$ In accordance with the recommendations of the Center for Migration Research of the Institute of Social Studies of the University of Warsaw, it was likewise assumed that the duration of migration in this case is of secondary importance [Górny A., Kaczmarczyk P. 2003: 9-10].

5 There are absolute and relative measures of migration. The most commonly used absolute migration meters include the net migration meter (migration balance). The relative measures of migratory traffic include the migratory influx (immigration) coefficient [Demografia 1984: 195-8]. An overview of absolute and relative measures of migration can be found in K. Woźniak's publication [2017: 142-4].

${ }^{6}$ The net migration meter (migration balance/pure migration) is the difference between population inflow and outflow from a specific area over a time period $t$. This can be described by the formula: $\mathrm{Mn}[\mathrm{t}]=\mathrm{N}[\mathrm{t}]-\mathrm{O}[\mathrm{t}]$ - where $\mathrm{Mn}[\mathrm{t}]$ - net migration in period $\mathrm{t}, \mathrm{N}[\mathrm{t}]$ - inflow [number of immigrants] in period $\mathrm{t}$; $\mathrm{O}[\mathrm{t}$ ] - outflow [number of emigrants] in period t [Demografia 1984: 197]. 


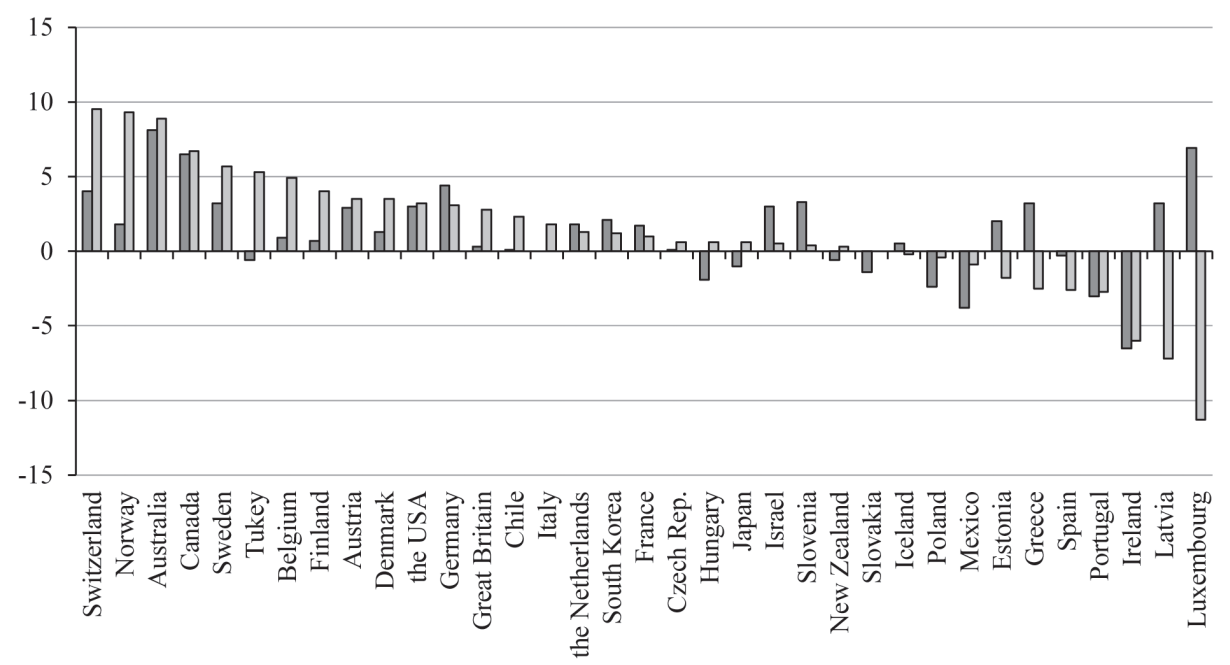

$\square 1990 \square 2015$

Figure 1. Net Migration in the OECD countries in 1990 and 2015 (per 1000 people) Explanations: The graph shows the net migration as the number of immigrants minus the number of emigrants in a given period, divided by the population in that period. The net migration was expressed per 1000 people.

Source: Own elaboration based on [Human Development Reports 2018; Net migration rate... 2019].

corded in Luxembourg (-11.3) and the highest in Switzerland (9.5). In some OECD countries there were also significant changes in the relationship between immigration and emigration. The majority of OECD countries had a positive net migration in the period under examination (meaning that in a given period, the amount of immigration exceeded the amount of emigration). The following countries had negative net migration, both in 1990 and 2015: Spain, Ireland, Mexico, Poland and Portugal. Moreover, in Japan, New Zealand and Hungary, in 2015, compared to 1990, the net migration turned from negative to positive. On the other hand, the following countries: Estonia, Greece, Luxembourg and Latvia had a positive net migration in 1990, whereas by 2015 the net migration was negative.

Figure 2 presents the inflow of immigrants for permanent and temporary residence to OECD countries between 1990 and 2016. It should be stressed, however, that in the case of several countries, the data are of a shorter time range (the details are below the chart). In absolute terms, the highest average inflow of immigrants in the years 1990-2016 was characteristic for USA and Germany. In turn, the lowest in this research period were Finland and Luxembourg. However, in relative terms, with respect 


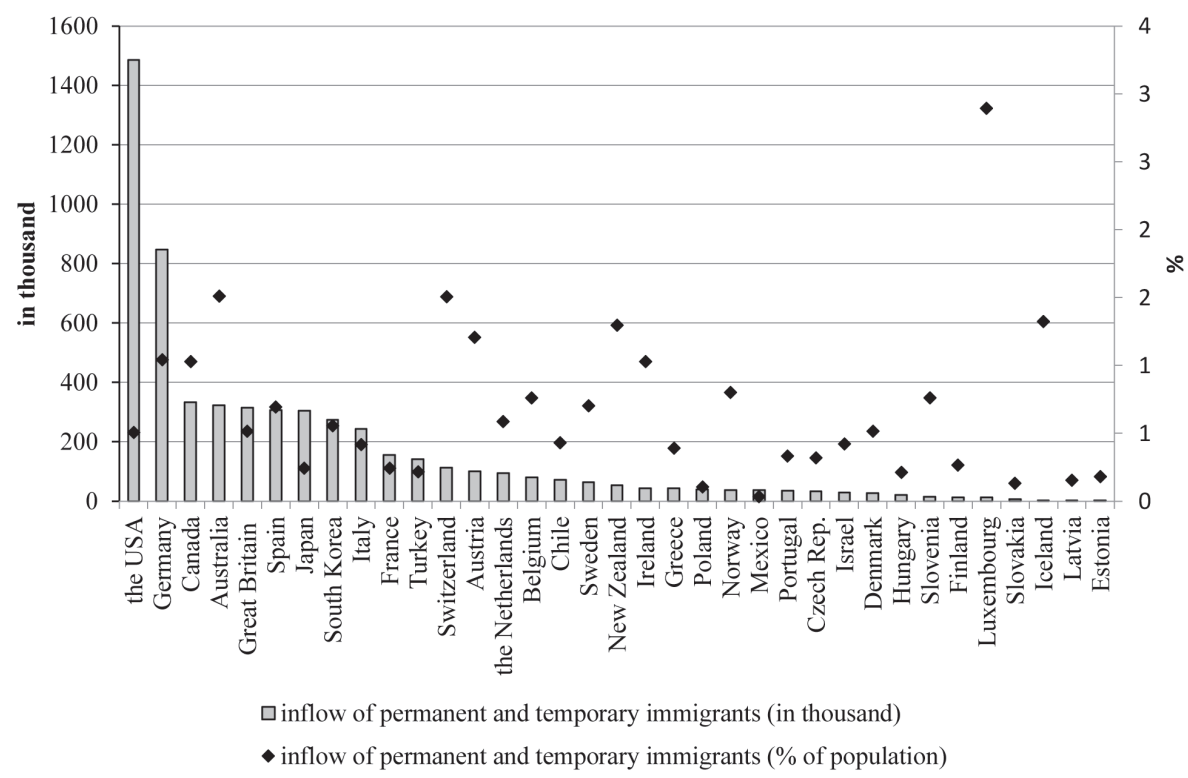

Figure 2: Inflows of immigrants to OECD countries for permanent and temporary residence between 1990 and 2016

Explanations: The graph shows the average values of the inflow of immigrants between 1990 and 2016. Due to lack of statistical data, the average was determined in the case of: Austria and Mexico for 1996-2016, Chile, South Korea: 2000-2016, Latvia: 2000, 2005-2016, Czech Republic: 1991, 1995-2016, Estonia: 2004-2016, Greece: 1998, 2005-2016, Israel, Poland, Slovenia: 1998-2016, Iceland: 1999-2016, Ireland: 1994-2016, Portugal: 1992-2016, Slovakia: 1993-2016, Turkey: 1995-2010. The inflow of immigrants was estimated based on the number of permits issued in a given period (for permanent and temporary residence) ${ }^{7}$.

Source: Own calculations based on International Migration Database... [2018].

${ }^{7}$ Since the so-called settlement, countries (Australia, Canada, Mexico and the USA) identify immigrants as those who have been granted permanent residence. Immigration data for these countries is divided into permanent and temporary immigration. However, for Australia and Canada permanent and temporary immigration data are only available for the years 2005-2015, while for 1990-2004 and 2016 - the number of temporary immigration inflows is assumed. Similarly, for Mexico, permanent and temporary immigration data are available only for the years 2005-2015, while for 1996-2004 and 2016 - temporary immigration flows are assumed. On the other hand, in the case of USA, permanent and temporary immigration data are only available for the years 2005-2016, while for the years 1990-2004, the number of temporary immigration inflow is assumed. In the case of Italy and Portugal, data for the entire research period include only temporary immigrants. 
to the population in a given country, the highest average inflow of immigrants was characteristic for the following countries: Luxembourg $(2.9 \%)$, Australia and Switzerland (1.51\% respectively).

The dynamics of the inflow of immigrants is also noteworthy. In most of the surveyed countries, the number of immigrants increased from year to year in the surveyed period. In many OECD countries, there was a decrease in the inflow of immigrants compared to previous years, especially after 2007, which may be related to the then financial crisis [Wiśniewski Z., Król A. 2014: 8, after: International Migration... 2012]. Between 1991 and 2016, the highest growth of the studied phenomenon was recorded in Portugal in 2001 compared to 2000. At that time, the inflow of immigrants increased nine fold. The greatest drop in the inflow was observed, in turn, at the turn of 1996-1997 in Mexico, where the 1997 inflow decreased by over $80 \%$ compared to the previous year. In most of the countries surveyed, however, there was an upward trend. In addition, the data presented indicate a significant diversity of OECD countries in terms of both the size of the inflow of immigrants (especially in relative terms) and the dynamics of the studied phenomenon.

\section{MIGRATION POLICY AS PART OF ECONOMIC POLICY}

Many definitions of economic policy can be found in both Polish and foreign language literature on the subject ${ }^{8}$. The vast majority of these definitions present economic policy as the influence of the state through specific tools and instruments on economic processes and phenomena. It should be stressed, however, that the concept of economic policy, although commonly used, cannot be considered as an unambiguous concept [Jarmołowicz W., Knapińska M. 2005: 78-82; Knapińska M., 2012: 90-1]. Consequently, taking into account different approaches to economic policy, it is worth pointing, first of all, to the problems of understanding economic goals presented by A. Horodecka [Knapińska M. 2012: 91-2 after: Horodecka A., 2008: 21]. The first of these approaches points to the normative approach, according to which economic policy goals come from values and are defined outside the system and in terms of norms characteristic of society. The second is a positive approach that emphasizes economic policy goals directly related to a specific economic deficit. The last,

\footnotetext{
${ }^{8}$ An overview of the definitions can also be found in: M. Knapińska [2012: 90-3].
} 


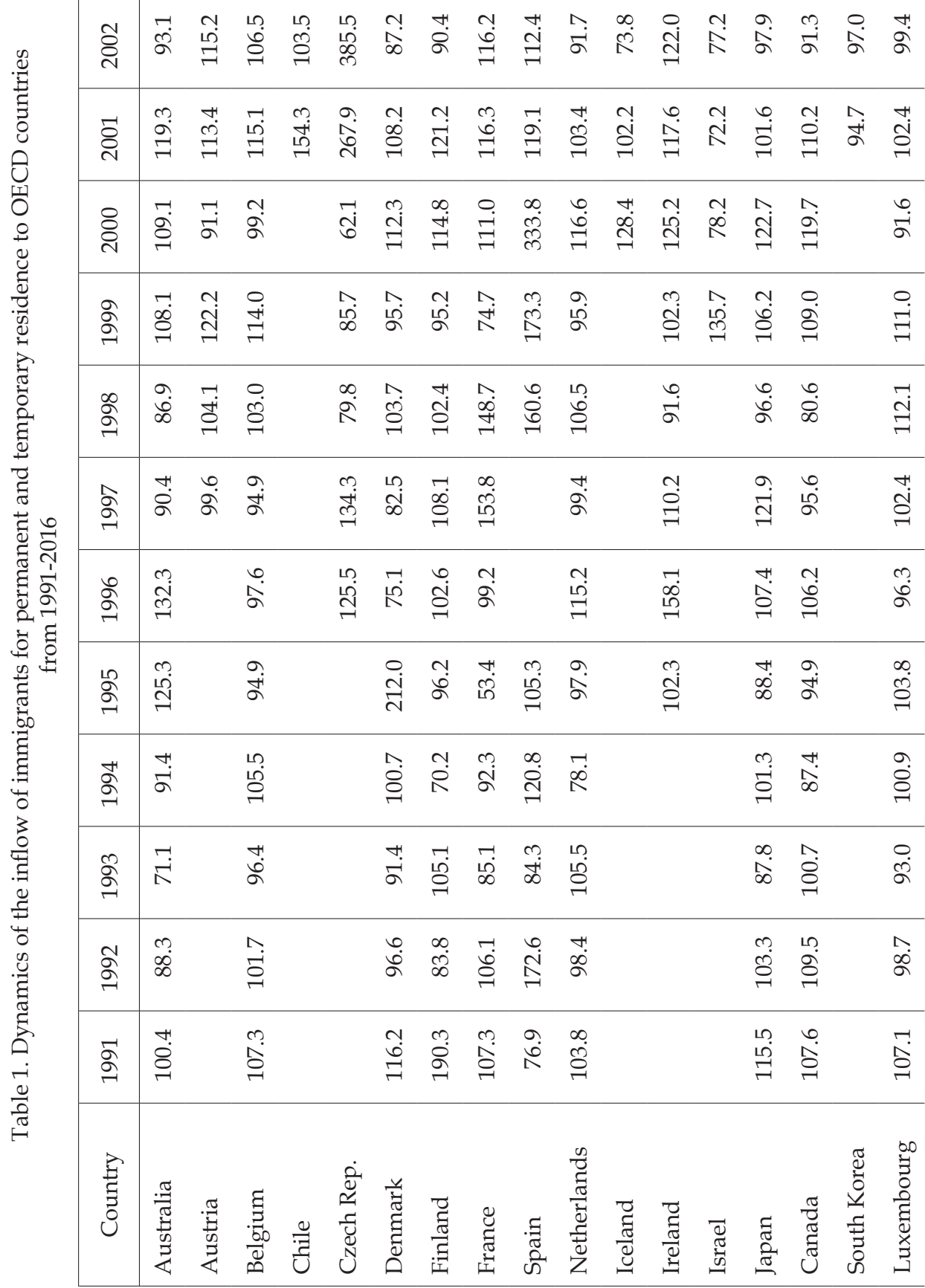




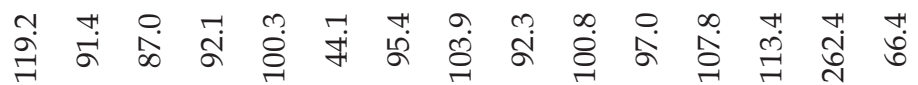

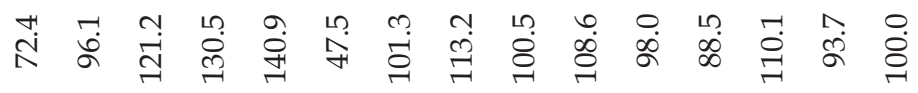

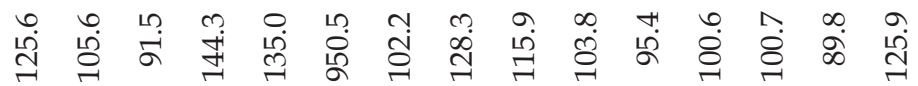

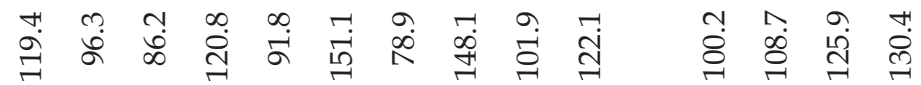

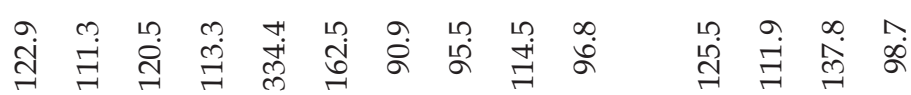

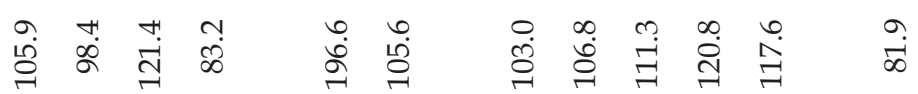

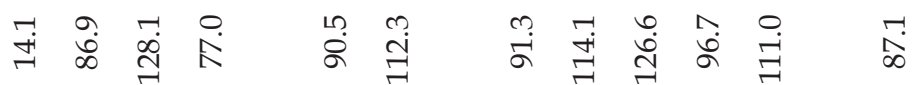

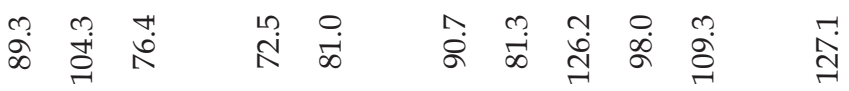

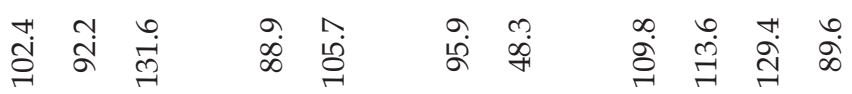

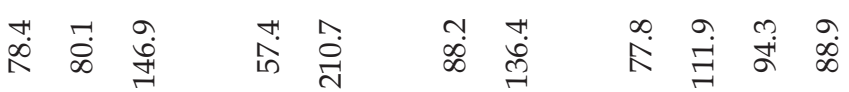

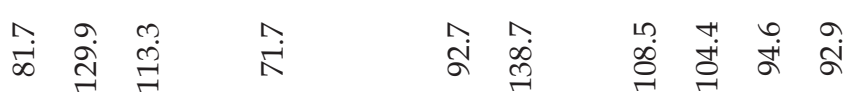

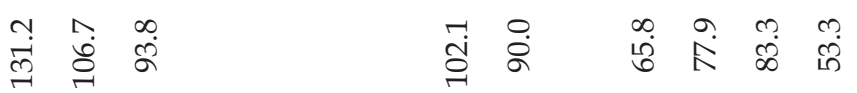

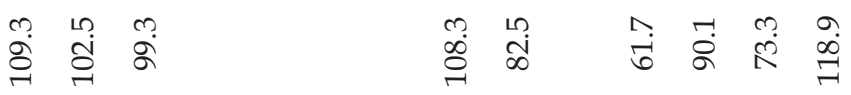

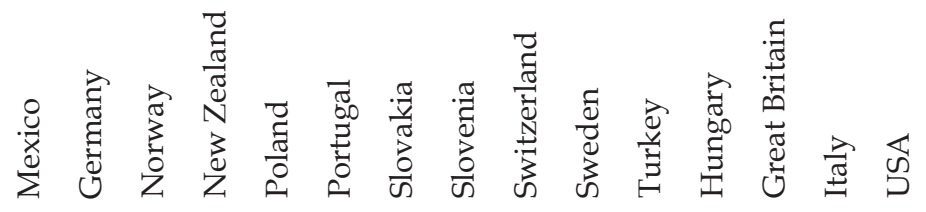




\begin{tabular}{|c|c|c|c|c|c|c|c|c|c|c|c|c|c|c|c|c|c|}
\hline $\begin{array}{l}0 \\
\stackrel{\sim}{\circ}\end{array}$ & $\begin{array}{l}\infty \\
\stackrel{\sim}{~}\end{array}$ & হి & ชู & $\begin{array}{l}\forall \\
\text { பீ }\end{array}$ & $\underset{\infty}{+\infty}$ & $\begin{array}{l}\text { Nִ } \\
ٍ \\
\end{array}$ & 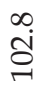 & $\begin{array}{l}0 \\
\dot{0} \\
\stackrel{0}{0}\end{array}$ & $\begin{array}{l}\text { ț } \\
\text { ลิ }\end{array}$ & $\begin{array}{l}m \\
\stackrel{2}{2}\end{array}$ & $\begin{array}{l}\text { m. } \\
\tilde{\omega} \\
\stackrel{n}{ }\end{array}$ & $\begin{array}{l}0 \\
\stackrel{\text { }}{\sim}\end{array}$ & $\underset{\stackrel{+}{+}}{\stackrel{+}{ت}}$ & $\stackrel{\text { ゙ָ }}{*}$ & $\begin{array}{l}+1 \\
\infty \\
10 \\
\end{array}$ & مे & $\begin{array}{l}3 \\
\stackrel{-}{0} \\
\stackrel{0}{0}\end{array}$ \\
\hline$\stackrel{\text { 뭉 }}{\stackrel{\sim}{\sim}}$ & ๙ุ & $\begin{array}{l}\infty \\
\infty \\
\stackrel{\sim}{0} \\
\end{array}$ & 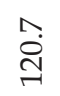 & $\begin{array}{l}0 \\
\text { ஸे } \\
\text { 음 }\end{array}$ & $\begin{array}{l}\widehat{N} \\
\text { ஸे }\end{array}$ & $\underset{\infty}{i}$ & $\begin{array}{l}\underset{7}{H} \\
\stackrel{7}{\sigma}\end{array}$ & 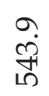 & $\begin{array}{l}0 \\
\dot{8}\end{array}$ & $\begin{array}{l}\infty \\
\infty \\
\propto\end{array}$ & $\begin{array}{l}\stackrel{\infty}{0} \\
\stackrel{\omega}{\sigma}\end{array}$ & $\begin{array}{l}\text { ઼ָ } \\
ٍ \\
ٍ\end{array}$ & $\begin{array}{l}\stackrel{+}{+} \\
\stackrel{+}{二}\end{array}$ & $\begin{array}{l}\widetilde{\sigma} \\
\text { ન્}\end{array}$ & $\underset{+}{\stackrel{+}{ت}}$ & $\begin{array}{l}\Lambda \\
\stackrel{10}{\sigma} \\
\stackrel{1}{\sigma}\end{array}$ & $\begin{array}{l}\text { ָै } \\
\text { ڤ̈ }\end{array}$ \\
\hline$\underset{\sim}{\stackrel{\sim}{\sigma}}$ & 우 & $\underset{+}{\stackrel{H}{+}}$ & 궁 & 웅 & $\begin{array}{l}+ \\
\stackrel{+}{+}\end{array}$ & $\begin{array}{l}N \\
\infty \\
\infty \\
\end{array}$ & $\begin{array}{l}\underset{0}{0} \\
\infty \\
\stackrel{\sigma}{\sigma}\end{array}$ & $\begin{array}{l}\stackrel{1}{i} \\
\infty\end{array}$ & ন̆ & $\hat{\sigma}$ & ঙ̛ & ஜூ & $\begin{array}{l}\stackrel{\sigma}{\oplus} \\
\stackrel{\oplus}{\sigma}\end{array}$ & 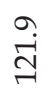 & $\begin{array}{l}0 \\
\stackrel{0}{0} \\
\stackrel{\sigma}{\sigma}\end{array}$ & $\begin{array}{l}\infty \\
\stackrel{+}{+}\end{array}$ & $\hat{\circ}$ \\
\hline 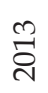 & $\stackrel{\text { gे }}{\text { g}}$ & 今ิ & స઼ & $\stackrel{0}{\stackrel{0}{R}}$ & $\begin{array}{l}\text { 옴 } \\
\stackrel{\text { N }}{\sim}\end{array}$ & م̊ & 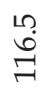 & $\stackrel{\stackrel{1}{\mathcal{F}}}{\stackrel{\mathrm{F}}{\sim}}$ & 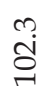 & $\begin{array}{l}\infty \\
\dot{0} \\
\dot{\sigma}\end{array}$ & $\begin{array}{l}\text { 우 } \\
\stackrel{0}{\rightleftharpoons}\end{array}$ & ન્ & 命 & $\begin{array}{l}\text { Nִ } \\
\stackrel{2}{\sim} \\
\end{array}$ & $\begin{array}{l}\overrightarrow{+} \\
\stackrel{2}{\sim}\end{array}$ & $\begin{array}{l}0 \\
\text { İ } \\
\text { Oे }\end{array}$ & $\stackrel{\varrho}{\varrho}$ \\
\hline 공 & $\underset{\sigma}{\sigma}$ & $\begin{array}{l}\stackrel{\oplus}{+} \\
\stackrel{+}{+}\end{array}$ & $\underset{\gamma}{0}$ & $\begin{array}{l}\infty \\
\text { م̂ }\end{array}$ & $\stackrel{\widehat{N}}{\stackrel{0}{\sim}}$ & $\begin{array}{l}+ \\
\infty \\
\infty \\
\sim\end{array}$ & તิ & 궁 & 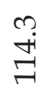 & $\begin{array}{l}0 \\
\infty \\
0 \\
0\end{array}$ & サூ & 머 & ડ̄ & $\begin{array}{l}m \\
2\end{array}$ & తి & $\begin{array}{l}\circ \\
\propto \\
\circ\end{array}$ & $\begin{array}{l}\stackrel{\vartheta}{\oplus} \\
\stackrel{\oplus}{\rightleftharpoons}\end{array}$ \\
\hline 공 & $\stackrel{+}{\stackrel{1}{\circ}}$ & $\begin{array}{l}\stackrel{+}{\oplus} \\
\stackrel{\oplus}{\sigma}\end{array}$ & 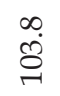 & مे & $\begin{array}{l}\stackrel{+}{~} \\
\stackrel{2}{\sigma}\end{array}$ & $\stackrel{\wedge}{N}$ & 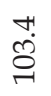 & $\begin{array}{l}\text { مे } \\
\text { ले }\end{array}$ & $\underset{\text { ㄱ }}{\stackrel{ }{ت}}$ & 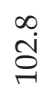 & బิ) & స్తి & 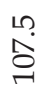 & $\begin{array}{l}\text { ○ } \\
\text { 子 } \\
\text { - }\end{array}$ & స̃ & 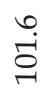 & $\stackrel{\circ}{2}$ \\
\hline 웅 & 궁 & 숭 & $\begin{array}{l}0 \\
\stackrel{0}{0} \\
\stackrel{\theta}{\sigma}\end{array}$ & $\begin{array}{l}a \\
\text { బa }\end{array}$ & $\begin{array}{l}\stackrel{0}{ } \\
\stackrel{1}{ } \\
\stackrel{\text { I }}{ }\end{array}$ & $\stackrel{H}{n}$ & $\begin{array}{l}0 \\
\stackrel{+}{0} \\
\stackrel{-}{1}\end{array}$ & $\begin{array}{l}\infty \\
\dot{\Gamma} \\
10\end{array}$ & 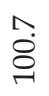 & 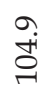 & $\stackrel{\curvearrowright}{\curvearrowright}$ & ஷ̊. & $\begin{array}{l}0 \\
\stackrel{0}{0} \\
\stackrel{0}{ }\end{array}$ & 강 & $\begin{array}{l}-\underset{\infty}{\infty} \\
\infty\end{array}$ & 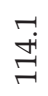 & $\begin{array}{l}0 \\
\stackrel{0}{\circ}\end{array}$ \\
\hline ஓे & $\begin{array}{l}\text { N̦ } \\
\text { ஸे }\end{array}$ & 공 & ڤ̊ & 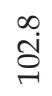 & $\begin{array}{l}\not{\infty} \\
\infty\end{array}$ & $\begin{array}{l}\text { Nִ } \\
\text { ז̊ }\end{array}$ & $\begin{array}{l}m \\
\infty \\
\infty\end{array}$ & 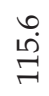 & ஷ̊ & $\begin{array}{l}\infty \\
\text { مू }\end{array}$ & \begin{tabular}{l}
10 \\
$\infty$ \\
$\infty$ \\
\hdashline
\end{tabular} & $\begin{array}{l}+ \\
\text { ti }\end{array}$ & 울 & $\begin{array}{l}\text { L? } \\
\text { மீ }\end{array}$ & $\begin{array}{l}\stackrel{+}{8} \\
\stackrel{2}{2}\end{array}$ & ț. & ઼ָ \\
\hline$\stackrel{\infty}{\stackrel{\sim}{~}}$ & 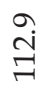 & 몽 & $\stackrel{\stackrel{1}{O}}{\stackrel{\oplus}{\rightleftharpoons}}$ & 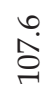 & ㄱ. & $\stackrel{m}{\mathbb{N}}$ & $\stackrel{\infty}{\stackrel{\infty}{~}}$ & $\begin{array}{l}\infty \\
\infty \\
\sigma^{\circ}\end{array}$ & $\begin{array}{l}\stackrel{\sim}{\oplus} \\
\stackrel{\sim}{\sigma}\end{array}$ & 궁 & $\begin{array}{l}\text { o } \\
\text { di }\end{array}$ & نَ & 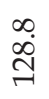 & $\stackrel{n}{\mathbb{N}}$ & 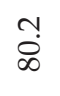 & $\begin{array}{l}0 \\
\stackrel{1}{1}\end{array}$ & $\underset{\sim}{\text { dె }}$ \\
\hline ڤ్) & $\begin{array}{l}\infty \\
\stackrel{\infty}{\circ} \\
\stackrel{\sim}{\sim}\end{array}$ & $\begin{array}{l}\not{O} \\
\stackrel{\circ}{\sigma}\end{array}$ & 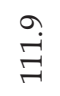 & 옹 & $\begin{array}{l}0 \\
\ddot{\sigma} \\
\sim\end{array}$ & $\begin{array}{l}0 \\
10 \\
10 \\
\stackrel{1}{1}\end{array}$ & 금 & $\frac{\text { Nִ }}{\stackrel{m}{\sigma}}$ & $\begin{array}{l}\text { ปै } \\
\text { ஸे }\end{array}$ & $\stackrel{4}{2}$ & $\stackrel{m}{n}$ & $\begin{array}{l}0 \\
\stackrel{+}{ \pm} \\
\stackrel{7}{-}\end{array}$ & $\begin{array}{l}0 \\
\infty \\
\rightarrow \\
=\end{array}$ & $\begin{array}{l}+ \\
\text { in } \\
\stackrel{-}{r}\end{array}$ & $\underset{\stackrel{\infty}{\vec{d}}}{\stackrel{\infty}{+}}$ & ت্t & $\stackrel{+}{\ddot{\sigma}}$ \\
\hline ঠి & 붕 & $\underset{+}{+}$ & 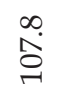 & $\stackrel{\nwarrow}{\stackrel{ }{\sim}}$ & ઼ָ & $\begin{array}{l}\widetilde{a} \\
\text { בี }\end{array}$ & $\begin{array}{l}\stackrel{0}{\circ} \\
\stackrel{\rho}{\sigma}\end{array}$ & 돔 & $\begin{array}{l}\infty \\
\infty \\
\infty \\
\infty\end{array}$ & $\begin{array}{l}\infty \\
\infty \\
0\end{array}$ & $\begin{array}{l}\infty \\
ٌ \\
0\end{array}$ & $\begin{array}{l}\stackrel{0}{~} \\
\stackrel{-}{\rightleftharpoons}\end{array}$ & స్ & مُ & 굴 & $\begin{array}{l}\circ \\
\text { бં }\end{array}$ & 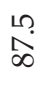 \\
\hline 용 & $\begin{array}{l}0 \\
\infty \\
0 \\
\varnothing\end{array}$ & $\stackrel{\circ}{\text { tí }}$ & $\begin{array}{l}a \\
\stackrel{\circ}{0}\end{array}$ & $\begin{array}{l}0 \\
\stackrel{2}{~} \\
\stackrel{1}{\sim}\end{array}$ & 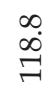 & $\begin{array}{l}n \\
\stackrel{2}{0} \\
F\end{array}$ & $\begin{array}{l}\text { 숭 } \\
\text { 음 }\end{array}$ & $\begin{array}{l}\text { ָุ } \\
\text { กิ }\end{array}$ & 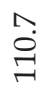 & $\begin{array}{l}0 \\
\text { ه̊ }\end{array}$ & & 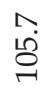 & సু & $\begin{array}{l}\text { 무 } \\
10 \\
\stackrel{10}{+}\end{array}$ & 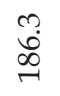 & 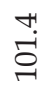 & 궁ㅁㅇ \\
\hline 苂 & $\begin{array}{l}\stackrel{ }{\infty} \\
\stackrel{-}{\sigma}\end{array}$ & 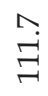 & 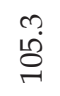 & 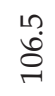 & $\begin{array}{l}0 \\
\stackrel{0}{0} \\
\end{array}$ & $\begin{array}{l}10 \\
\infty \\
\infty\end{array}$ & 궁 & & $\begin{array}{l}\stackrel{0}{\text { I }} \\
\text { ㄱ. }\end{array}$ & $\begin{array}{l}\infty \\
\dot{8} \\
\dot{\sigma}\end{array}$ & & 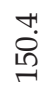 & $\begin{array}{l}10 \\
\infty \\
\infty\end{array}$ & $\begin{array}{l}0 \\
\infty \\
\sigma\end{array}$ & $\begin{array}{l}\wedge \\
\stackrel{1}{\infty} \\
\infty\end{array}$ & $\begin{array}{l}\infty \\
\infty \\
\infty\end{array}$ & டீ? \\
\hline 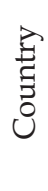 & 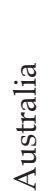 & 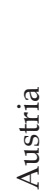 & 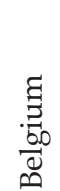 & $\frac{\tilde{\sigma}}{\tilde{\sigma}}$ & $\frac{\vartheta}{\stackrel{\Xi}{U}}$ & 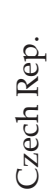 & 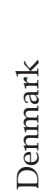 & 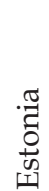 & $\begin{array}{l}\widetilde{\Xi} \\
\underset{\Xi}{\Xi} \\
\stackrel{\Xi}{ \pm}\end{array}$ & 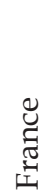 & 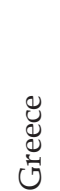 & $\begin{array}{l}\text { ే } \\
\text { के }\end{array}$ & 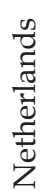 & $\begin{array}{l}\overrightarrow{\widetilde{E}} \\
\frac{\vec{d}}{\mathbb{d}} \\
\end{array}$ & 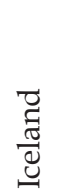 & $\begin{array}{l}\vec{g} \\
\tilde{\sigma} \\
\omega\end{array}$ & $\begin{array}{l}\tilde{\Xi} \\
\text { हే } \\
.\end{array}$ \\
\hline
\end{tabular}




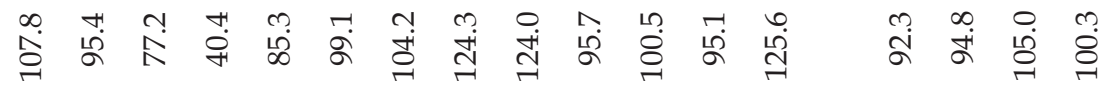

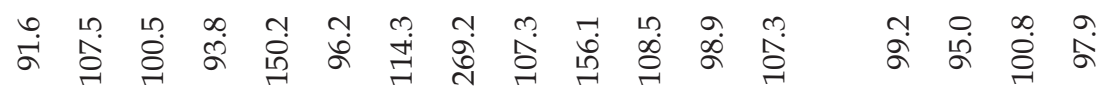

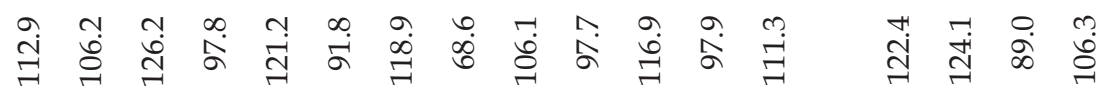

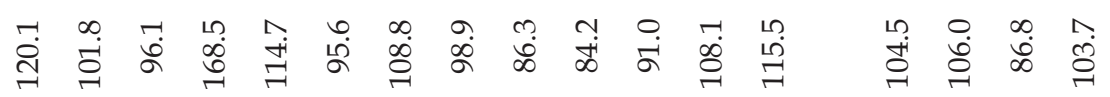

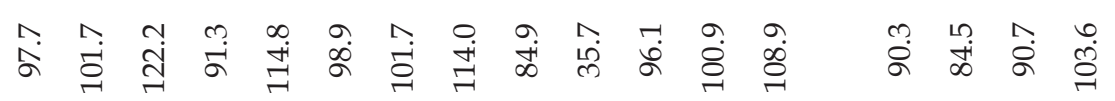

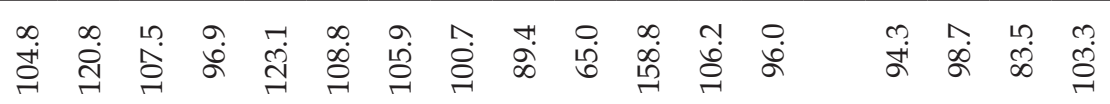

윰

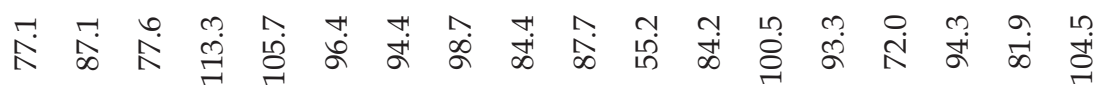

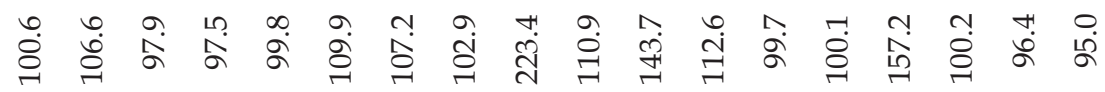

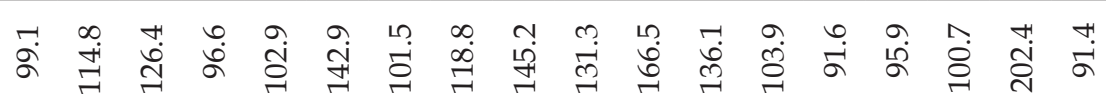

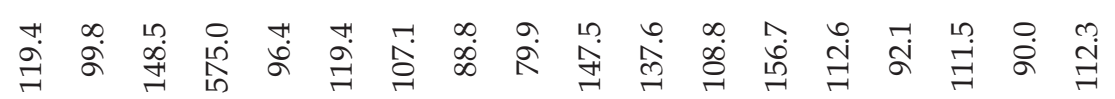

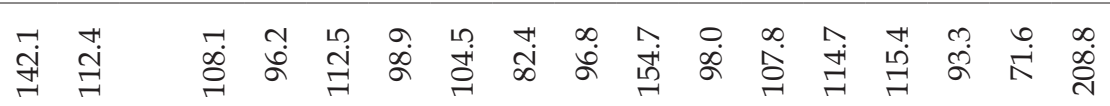

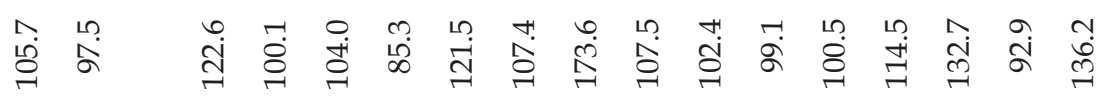

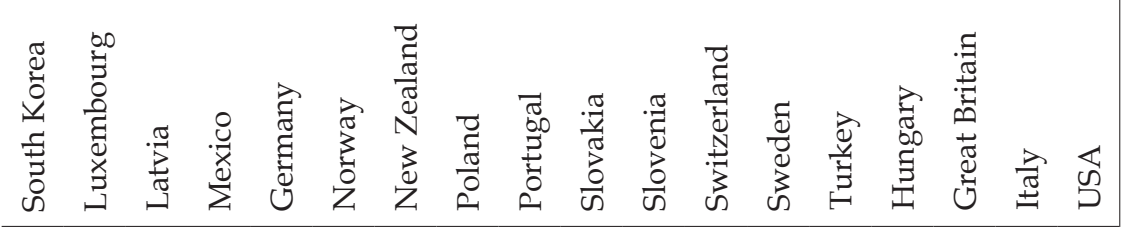


descriptive approach indicates the objectives actually formulated by economic policy makers at a given time and place [Knapińska M. 2012: 91-2 after: Horodecka A., 2008: 21].

One of the essential parts of economic policy is migration policy. This relationship is justified in particular by the fact that the migration policy is an instrument necessary to achieve the objectives of economic policy, and also by the fact that migration policy is a part of the state's demographic development policy [Kocan V., 2005: 85, after: Olszewska, 2005: 34]. In a broad sense, migration policy is, therefore, an extension of economic policy and the main role in shaping it is played, not only by laws and norms, but also by interest groups [Lesińska M., 2011: 5].

The history of migration flows also played an important role in shaping the migration policies of nations. After the Second World War, most European countries actively recruited foreign labour to rebuild their economies, which had been destroyed by the war. Until the mid-1970s, circular migration, in particular, developed between the migrant's country of origin and the host country, in which migrants took up employment in the host country without any intention of settling there permanently. Examples of circular migration at that time are migration flows between North Africa and Europe through the Sicilian Strait and between France and migrants from Senegal, Portugal and Morocco. With the recession (1974) following the oil crisis, countries that had so far recruited foreign labour have mostly closed their borders to further inflows of labour migrants. This change led to a sharp increase in the number of illegal migrants, while at the same time encouraging an increase in family reunification and the settlement of foreigners who were considered to be the most "problematic". In view of the migration flows described above, it can therefore be concluded that migration policies were formulated gradually, but often based on incorrect forecasts. One example is the fall of the Berlin Wall in 1991, which lifted political barriers to migration flows. Contrary to many fears, however, this did not result in a mass influx of immigrants. Similarly, the gradual opening of borders by the countries of Central and Eastern Europe led, for the most part, to circular migration, which was not accompanied by permanent settlement in the host country. History has shown that migration policies, in many cases, have not been relevant to the actual migration flows or the aspirations of migrants [Bassi M. 2017].

The report entitled "Migration Governance Framework" developed by the International Organization for Migration indicates that migration policy includes laws and policies related to the movement of people. It also 
deals with the following issues: travel and temporary mobility, immigration, emigration, nationality, labour markets, socio-economic development, industry ${ }^{9}$, trade, social cohesion, social services, health, education, law enforcement, foreign policy, trade and humanitarian issues [Migration Governance... 2019]. In the context of analysing migration policy from an economic policy point of view, it should also be noted that many other policies, which are not seen as part of migration policy can have an impact on migration, for example: labour market policy, social policy, education policy. H. de Haas and M. Czaika [2013: 489] emphasize that the differentiation of individual policies is connected with many difficulties, and even if the "border" can be drawn, the regulations of other policies (for instance, those related to the labour market) can influence the migration of people. The relationship between migration policy and other economic policies should, therefore, be regarded as particularly important. Furthermore, the analysis of the links between economic and political change is considered crucial in the development of migration policy. On the one hand, immigration policy is a response to political and economic change and on the other hand, migration flows are a response to policy changes. The analysis of these links is therefore crucial in the development of migration policies [Bodvarsson Ö.B. et al. 2015: 45]. In the context of the process of an ageing population ${ }^{10}$ (considered to be one of the greatest challenges for the functioning of the labour market), the important role of migration policy is also seen in the process of mitigating the decline in labour supply [Wiśniewski Z. 2018: 275, 277; Wiśniewski Z., Maksym M. 2018: 23-5].

The border control and related - illegal migration is also one of the key areas of managing migration flows within the framework of migration policy. Among the reasons that hinder effective management of illegal immigration, it is especially pointed out that developed countries need to take simultaneous actions to integrate immigrants, minimize social costs and maximize economic benefits. Despite many difficulties in managing illegal migration, different countries implement different measures and take different actions to prevent illegal migration. In the context of illegal

${ }^{9}$ M. Słodowa-Hełpa emphasizes that social and economic development refers to the development mechanism, in which economic development determines social development, and vice versa [Słodowa-Hełpa M. 2013: 30].

${ }^{10}$ In the context of the phenomenon of population ageing, the need to take into account the issue of demographic change in the planning of employment by companies, in particular through appropriate ways of recruiting older people is also stressed [Jarecki W. 2014: 146-50]. 
migration, it is particularly stressed that developed countries should implement changes in migration policy - i.e. respond to the illegal influx of economic migrants and, furthermore, to the influx of asylum seekers (inter alia, through incentives or penalties for immigrants, sanctions, border control measures). [Bhagwati J.N. 2003: 102-3].

\section{MIGRATION POLICY ON BORDER CONTROL IN OECD COUNTRIES}

The literature on the subject emphasizes that illegal immigration is, by its very nature, an immeasurable phenomenon, as it is difficult to calculate the exact number of people with unregulated status. The sources of data on the scale of this phenomenon, in particular, can be data obtained during police abolition (a situation where illegal immigrants report to the authorities of a given country for regularisation). This also includes police activities, interrogation of migrants at the country's borders, as well as indepth research conducted by institutions, NGOs and researchers within the population of a given country.[Thiollet H. 2017: 31-2].

This section attempts to assess the capacity of OECD countries to enforce border control regulations. It should be stressed, however, that the selection of variables for the field analysis of border control enforcement has been associated with many difficulties, particularly, the lack of comparable statistical data for individual OECD countries. Difficulties in the selection of statistical data were also related to diverse methodology adopted in different statistical data sources. The most reliable statistical data relating to this area of migration policy were those obtained from the European Statistical Office (Eurostat) ${ }^{11}$, which, however, were not available to all the countries belonging to the Organization for Economic Cooperation and Development.

${ }^{11}$ With regard to the European Union, it should also be stressed that the action programme to combat illegal immigration was adopted by the Council of the European Union in 2002. In turn, the European Parliament in 2008 adopted a Directive on common standards and procedures in Member States for returning third-country nationals illegally staying. The Directive also defines the general conditions for returning illegal foreigners [Lecadet C. 2017: 47]. 
Among these data, four variables can be distinguished, including the number of identified third-country nationals ${ }^{12}$ illegally staying in particular countries; the number of third-country nationals refused entry at the border; the number of third-country nationals who were ordered to leave the country (by gender, nationality and age); and the number of thirdcountry nationals that were returned to their country of origin after being ordered to leave the country (by gender, nationality and age. This, however, depends on the assistance received, country of origin and procedures such as re-admission agreements). These variables are presented in Table 2.

Table 2. Variables describing the effectiveness of migration policy in the area of border control

\begin{tabular}{|c|c|c|c|}
\hline $\begin{array}{c}\text { Variable } \\
\text { designation }\end{array}$ & Variable characteristics & $\begin{array}{l}\text { Variable } \\
\text { character }\end{array}$ & Source \\
\hline Illegal & $\begin{array}{l}\text { Identified third-country nationals illegally staying } \\
\text { in the country }\end{array}$ & - & \multirow{5}{*}{ 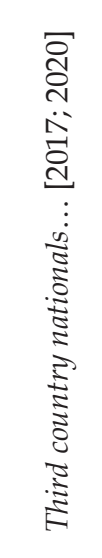 } \\
\hline Order & $\begin{array}{l}\text { Third country nationals who were ordered to leave } \\
\text { the country }\end{array}$ & - & \\
\hline Refusal & $\begin{array}{l}\text { Third country nationals refused entry at the national } \\
\text { border }\end{array}$ & - & \\
\hline Returned & $\begin{array}{l}\text { Third country nationals who were returned to their } \\
\text { country of origin after being ordered to leave the } \\
\text { country }\end{array}$ & - & \\
\hline $\begin{array}{l}\text { Returned } \\
\text { percentage }\end{array}$ & $\begin{array}{l}\text { Third-country nationals who were returned to } \\
\text { their country of origin after being ordered to leave } \\
\text { [\% of third country nationals who were ordered to } \\
\text { leave] }\end{array}$ & stimulant & \\
\hline
\end{tabular}

Source: Own study.

However, given that the first two variables represent the phenomenon of illegal immigration, they do not allow the assessment of a country's ability to enforce migration policy in this area, as it is impossible to relate each of them to the total number of illegal third-country nationals in that country and the total number of third-country nationals who

12 A third-country national is a person who is not a citizen of the European Union within the meaning of Article 20(1) of the Treaty on the Functioning of the European Union and who is not a person enjoying the right of free movement under EU law pursuant to Article 2(5) of Regulation (EU) 2016/399 on the Union Code on the rules governing the movement of persons across borders (Schengen Borders Code) [Migration.... 2018]. 
have attempted to enter the country illegally. Only the comparison of the other two variables, i.e., the reference to the number of third-country nationals deported to their country of origin after receiving an order to leave the country to the number of third-country nationals who were issued with this injunction allowed to obtain a variable, necessary for the analysis.

According to the research conducted to date, indicating in particular that the inflow of illegal immigrants may have a negative impact on the economy, mainly due to the development of a "shadow economy" and the increase in the burden of the tax system in host countries [Hanson G.H. 2009], this variable has been classified as a stimulus.

The first variable, i.e. third-country nationals who have been declared by the authorities of a Member State to be illegally staying, refers to persons who entered the country illegally (for example, by avoiding immigration controls or holding false documents that were not previously detected) or persons who lost their residence permit while still remaining in the country [Enforcement of Immigration... 2018].

Although this variable provides some information on the number of illegal immigrants (at least those identified by the authorities of a given country), it does not allow it to be included in the assessment of the border control enforcement capacity of OECD countries. For this to be possible, this variable would need to refer to the number of illegal third-country nationals in the country in question. In that case, it would be possible to examine how many illegal immigrants have been identified in a given country, which in turn would give the ability to assess the effectiveness of the actions taken. However, such data related to the nature of the phenomenon of illegal migration are not available as was stressed earlier.

A similar situation applies to another variable - the number of thirdcountry nationals who were formally refused entry at the national border. The grounds for refusing entry into the country may include, among others, the lack of a valid travel document, possession of forged/counterfeit/ falsified travel documents, recognition of a person as a threat [Migration and Home... 2018; Enforcement of Immigration... 2018].

Although this variable provides information on how many people were turned back at the border, however that does not guarantee its inclusion in the assessment of the border control enforcement capacity of OECD countries. Such an assessment would only be possible if data on how many people actually tried to cross the border illegally were available. The comparison of the other two variables i.e. the number of third-country nation- 
als who were ordered to leave a country and the number of third-country nationals who left a country after being ordered to do so makes it possible to examine the capacity of OECD countries to enforce border control rules by determining the percentage of third-country nationals actually leaving the country after being ordered to leave its territory.

The basic statistics of these variables are presented in Table 3. Between 2008 and 2016, the average number of third-country nationals who were found illegally staying by the authorities of a Member State was 32.43 thousand people. In the years under study, a smaller number of third-country nationals were ordered to leave the country (22.36 thousand persons). In turn, the average number of third country nationals who were formally refused entry at the border between 2008 and 2016 was 16.21 thousand people. The lowest number of third-country nationals, 9.48 thousand people, left the country after receiving an order in the examined period.

Between 2008 and 2016, the average percentage of third-country nationals who actually left the country after being ordered to leave was $52.8 \%$. Moreover, the highest variation with respect to the discussed variables was observed among the surveyed countries in the number of thirdcountry nationals who were formally refused entry at the national border (the coefficient of variation between 2008 and 2016 was 355\%).

Table 3. Descriptive statistics of variables in the area of border control ( $x 1000)$

\begin{tabular}{|c|c|c|c|c|c|c|c|c|c|c|}
\hline Variable & Min & $Q_{1}$ & $Q_{2}$ & M & $Q_{3}$ & Max & $S D$ & $\begin{array}{l}\mathrm{CV} \\
{[\%]}\end{array}$ & 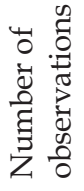 & 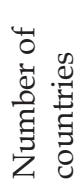 \\
\hline Illegal & 0.13 & 1.66 & 6.66 & 32.43 & 42.08 & 911.47 & 82.08 & 253 & 207 & 23 \\
\hline Order & 0.11 & 2.11 & 9.91 & 22.36 & 31.71 & 146.34 & 29.17 & 130 & 207 & 23 \\
\hline Refusal & 0.00 & 0.54 & 2.06 & 16.21 & 7.68 & 510.01 & 57.51 & 355 & 207 & 23 \\
\hline Returned & 0.08 & 0.87 & 4.42 & 9.48 & 11.23 & 75.82 & 14.43 & 152 & 207 & 23 \\
\hline $\begin{array}{l}\text { Returned } \\
\text { percentage }\end{array}$ & 6.50 & 25.1 & 50.9 & 52.8 & 75.40 & 208.5 & 30.80 & 59 & 207 & 23 \\
\hline
\end{tabular}

Explanations: Min - minimum value; $Q_{1}$ - first quartile; $Q_{2}$ - second quartile; $M$ - arithmetic mean; $Q_{3}$ - third quartile; Max - maximum value; $S D$ - standard deviation; $C V$ [\%] - coefficient of variation in percentage.

Source: based on own study, Third country nationals... [2017, 2020]. 


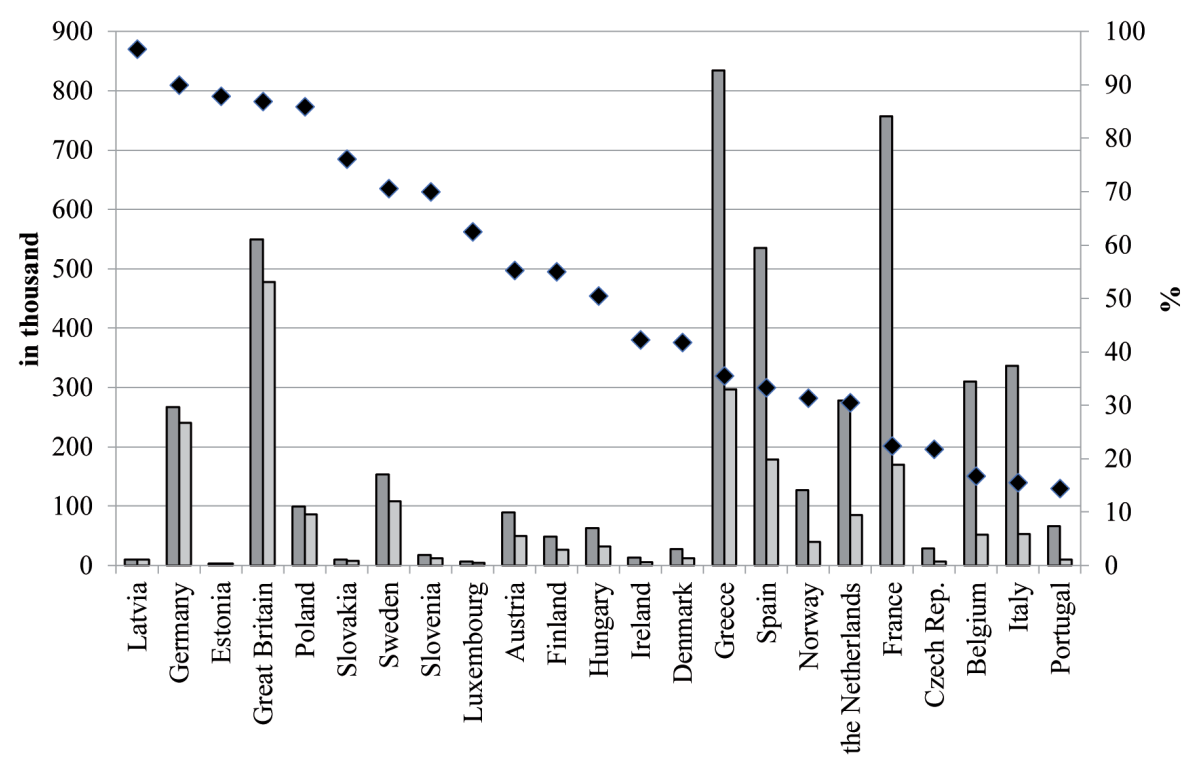

$\square$ Third country nationals ordered to leave (in thousand)

$\square$ Third country nationals returned following an order to leave (in thousand)

- The percentage of third-country nationals who left the country after the order was issued

Figure 3. Third-country nationals who were issued with an order to leave and who actually left the country after the order was issued between 2008 and $2016^{13}$

Explanation: The chart shows the sum of third-country nationals who were issued an order to leave the country and who actually left the country after the order was issued by the country between 2008 and 2016.

Source: Own study based on Third country nationals... [2017, 2020].

${ }^{13}$ In order to maintain the consistency of empirical analyses carried out in the scope of the scale and dynamics of population immigration, as well as in the scope of illegal migration, the calculation of irregular migration includes data until 2016 (the limit of the availability of statistical data on the basis of which the calculation of the scale and dynamics of population immigration was done is 2016). In order to verify the obtained results, an empirical analysis was carried out for the years 2008-2019. The obtained results are consistent with the results obtained for the years 2008-2016, the highest percentage of third-country nationals who were issued an order to leave the country and who actually left its territory after this order was issued were characterized by Latvia (96.4\%), Estonia (89.4\%), Great Britain (89.6\%) and Germany (74.9\%). A slight increase in the level of law enforcement with respect to border control, measured by the percentage of third-country nationals who left the territory of a given country after receiving the warrant, was also noted for Slovenia $(120.2 \%)$ and Slovakia (77.9\%) [based on own calculations: Third country nationals... 2020]. Therefore, taking into account the latest available data on irregular migration for 2017-2019 
Figure 3 shows the percentage of third-country nationals who actually left the territory of a country after being ordered to leave it. As mentioned earlier, comparable data are available in this case only for the Member States of the European Union between 2008 and 2016. Between 2008 and 2016, in total, the following countries issued the largest number of exit orders: Greece, France, Great Britain, Spain, Italy, Belgium, Germany and the Netherlands. These countries were characterized by a varied level of enforcement of the execution of orders issued by third-country nationals. Between 2008 and 2016, Germany and the United Kingdom had the highest percentage of third-country nationals who were issued an order and actually left the territory after the issuance of an exit order. From the data presented in Figure 3, it can also be concluded that the remaining countries with relatively more exit orders were characterised by a significantly lower percentage of third-country nationals leaving the territory after the order was issued.

However, the data presented in Figure 3 should be approached with caution, since the number of third-country nationals leaving a given country between 2008 and 2016 may result from an order to leave the territory of a given country even before 2008 . Then it could turn out that the law enforcement in this case is less respected than the data presented in the graph indicates. However, data before 2008 is unavailable.

\section{CONCLUSIONS}

Empirical analyses in the field of population migration (including immigration and emigration) are particularly dependent upon the adopted research perspective as well as the availability of statistical data used in carrying out these analyses. Comparison of national statistics on international migration is associated with many difficulties at the global level. Countries use different concepts and definitions to define migrants. The schedule of statistical data collection in this area is also different. Hence, reference should be made, in particular, to statistical data developed by inter-

in this analysis does not significantly affect the results obtained. The obtained results may also suggest that migration policy in the area of border control of the above-mentioned countries has become more restrictive. However, as in the case of the analysis for the years 2008-2016, the presented data should be approached with caution, as the number of thirdcountry nationals leaving a given country in the years 2008-2019 may result from an order to leave the territory of the country before 2008 . 
national organizations. Based on the analyses of statistical data published by international organizations, for the purpose of quantitative analysis of the scale and dynamics of population immigration in OECD countries, the Organization for Economic Cooperation and Development definition was adopted, according to which population immigration was estimated based on the number of permits issued during a given period (permanent and temporary residence) [International Migration Database... 2018].

Migration policy as part of economic policy is an instrument necessary to achieve the objectives of economic policy in many important areas of state activity. Moreover, regulations on other policies (for example, those related to the labour market) can also influence the migration of the population. The relationship between migration policy and other economic policy areas should therefore be regarded as particularly important. On the basis of the analysis of the scale and dynamics of population migration in the OECD countries, it can be concluded that in most of the countries under study, the number of immigrants increased from year to year in the given period. The presented data also indicate a significant diversity of OECD countries in terms of both the size of the inflow of immigrants (especially in relative terms) and the dynamics of the studied phenomenon. Therefore, both the scale and dynamics of population migration in individual countries have a significant impact on the development of migration policies, as well as other related policies.

With regard to the analysis of the capacity of OECD countries to enforce border control regulations, it was indicated that between 2008 and 2016, the following countries issued the highest number of exit orders: Greece, France, the United Kingdom, Spain, Italy, Belgium, Germany and the Netherlands. These countries were characterized by varying levels of enforcement of the orders issued and compliance by third-country nationals. By contrast, Latvia, Estonia, Germany and the United Kingdom had the highest percentage of third-country nationals who were issued with orders to leave the country and actually left the territory between 2008 and 2016 after the order was issued. The available data, however, do not allow for detailed conclusions to be drawn with regard to the enforcement capacity of OECD countries in the field of border control, but only allow for some general trends in this respect. The inability to draw specific conclusions in this area is due, in particular, to the very nature of illegal immigration, which is a difficult phenomenon to measure.

Among the reasons that hinder effective management of illegal immigration, the necessity for developed countries to take simultaneous ac- 
tions for integrating immigrants, minimizing social costs and maximizing economic benefits has, particularly, been pointed out. Despite many difficulties in managing illegal migration, different countries implement different measures and take different actions to prevent illegal migration. In the context of illegal migration, it is mainly stressed that developed countries should implement changes in migration policy - i.e. respond to the illegal influx of economic migrants and. consequently, the influx of asylum seekers (inter alia, through incentives or penalties for immigrants, sanctions, border control measures). [Bhagwati J.N. 2003: 102-3]. Studies so far also indicate that the factors that attract migrants to a given country have a more positive impact on migration as the immigration laws of the receiving country become less restrictive [Mayda A.M. 2010]. In the framework of further research, it seems appropriate to make an empirical analysis, bearing in mind the factors determining migration policies of individual countries, especially in the context of the diversity of countries in terms of scale and dynamics of population immigration.

\section{REFERENCES}

\section{Literature}

Bassi M. (2017), Czy wszyscy imigranci chca zamieszkać w Europie na stałe? [in:] Thiollet H. (ed.), Migranci, migracje. O czym warto wiedzié, by wyrobić sobie własne zdanie, Armand Colin, Malakoff, Kraków, 36-39.

Bhagwati J.N. (2003), Borders Beyond Control, Foreign Affairs, 82: 98-104.

Bodvarsson Ö.B., Simpson N.B., Sparber C. (2015), Migration Theory [in:] Chiswick B., Miller P. (eds.), Handbook of the Economics of International Migration: The Immigrants, Elsevier B.V., Amsterdam, 3-53.

Castles S., Miller M.J. (2011), Migracje we wspótczesnym świecie, Wydawnictwo Naukowe PWN, Warszawa.

Chapman M., Prothero M. (1985), Themes on Circulation in Third World [in:] Chapman M. (ed.), Circulation in the Third World Countries, Rouledge \& Kegan Paul, London/ Boston/Melbourne.

de Arce R., Mahia R. (2008), Determinants of Bilateral Immigration Flows Between The European Union and some Mediterranean Partner Countries: Algeria, Egypt, Morocco, Tunisia and Turkey, MPRA Working Paper, 14547, Munich Personal RePEc Archive, Munich.

de Haas H., Czaika M. (2013), The Effectiveness of Immigration Policy, Population and Development Review, 39(3), 487-508.

Demografia. Metody i analizy prognozowania, (1984), Cieślak M. (ed.), Państwowe Wydawnictwo Naukowe, Warszawa.

Górny A., Kaczmarczyk P. (2003), Uwarunkowania i mechanizmy migracji zarobkowych w świetle wybranych koncepcji teoretycznych, Prace Migracyjne, 49, 1-92.

Grau Grau A.J., Ramírez López F. (2017), Determinants of Immigration in Europe. The Relevance of Life Expectancy and Environmental Sustainability, Sustainability, nr 9: 1-17. 
Hanson G.H. (2009), The Economics and Policy of Illegal Immigration in the United States, Migration Policy Institute, Washington.

Horodecka A. (2008), Ewolucja celów polityki gospodarczej. Rola zmian otoczenia, Wydawnictwo Naukowe PWN, Warszawa.

Hugo G. (1982), Circular Migration in Indonesia, Population and Development Review, 8(1), 59-83.

Jarecki W. (2014), Rekrutacja pracowników starszych w kontekście zmian demograficznych. Analiza na przykładzie województwa zachodniopomorskiego, Zeszyty Naukowe Uniwersytetu Szczecińskiego. Ekonomiczne Problemy Ustug, 114, 143-151.

Jarmołowicz W., Knapińska M. (2005), Polityka państwa na rynku pracy w warunkach transformacji gospodarczej. Akademia Ekonomiczna w Poznaniu, Poznań.

Kaczmarczyk P. (2002), Mobilność cyrkulacyjna jako kategoria badawcza w naukach społecznych, Studia Socjologiczne, 167(4), 37-66.

Knapińska M. (2012), Wspólny europejski rynek pracy. Geneza - rozwój - funkcjonowanie, Wydawnictwo Uniwersytetu Ekonomicznego w Poznaniu, Poznań.

Kocan V. (2005), Wspótczesna migracja i polityka migracyjna na przykładzie Hiszpanii, Warmińsko-mazurski Kwartalnik Naukowy, Nauki Społeczne, 2, 77-96.

Lecadet C. (2017), Czy wydalenie cudzoziemców to w demokracji normalna praktyka? [in:] Thiollet H. (ed.), Migranci, migracje. O czym warto wiedzieć, by wyrobić sobie wtasne zdanie, Armand Colin, Malakoff, Kraków, 46-48.

Lesińska M. (2011), Migracje we wspótczesnej analizie politologicznej: Niewykorzystany potencjat, CMR Working Papers, 47(105), 1-32.

Massey S.D., Arango J., Hugo G., Kouaouci A., Pellegrino A., Taylor J.E. (1993), Theories of International Migration: A Review and Appraisal, Population and Development Review, 19(3): 431-466.

Mayda A.M. (2010), International migration: a panel data analysis of the determinants of bilateral flows, Journal of Population Economics, 23(4): 1249-1274.

Okólski M., Fihel A. (2012), Demografia. Wspótczesne zjawiska i teorie, Wydawnictwo Naukowe Scholar, Warszawa.

Olszewska O. (2005), Prawo jako instrument polityki migracyjnej - kształtujący czy wspomagający, [in:] Iglicka K., Olszewska O., Stachurski A., Murawska J., Dylematy polityki migracyjnej Polski, Serie Prace Migracyjne, 58, Uniwersytet Warszawski, Instytut Studiów Społecznych, Warszawa, 34-39.

Słodowa-Hełpa M. (2013), Rozwój zintegrowany. Warunki, wymiary, wyzwania, CeDeWu, Warszawa.

Thiollet H. (2017), Czy w Europie mamy do czynienia z ogromna nielegalna imigracją? [in:] Thiollet H. (ed.), Migranci, migracje. O czym warto wiedzieć, by wyrobić sobie własne zdanie, Armand Colin, Malakoff, Kraków, 31-35.

Wiśniewski Z. (2018), Aktywność zawodowa i formy zatrudnienia w Polsce, Prace Naukowe Uniwersytetu Ekonomicznego we Wrocławiu, 511, 269-278.

Wiśniewski Z., Maksim M. (2018), Sytuacja i aktywizacja zawodowa bezrobotnych 50 plus - doświadczenia niemieckie, Problemy Polityki Społecznej, 3, 23-36.

Wiśniewski Z.. Król A. (2014), Emigranci z Polski na norweskim rynku pracy, Polityka społeczna, 4, 8-13.

Woźniak K. (2017), Zróżnicowanie natężenia zagranicznych migracji ludności (na przykładzie województw w Polsce), Studia Oeconomica Posnaniensia, 5(5), 139-157. 


\section{Statistics sources}

Colombia, (2020), Organisation for Economic Co-operation and Development [OECD], https://www.oecd.org/colombia/, accessed 23.07.2020.

Database on Immigrants in OECD and non-OECD Countries: DIOC, (2018), Organisation for Economic Co-operation and Development [OECD], http://www.oecd.org/els/mig/ dioc.htm, accessed 23.07.2020.

DEMIG POLICY data, (2020), International Migration Institute, https://www.imi.ox.ac. uk/data/demig-data/demig-policy-1, accessed 23.07.2020.

Enforcement of Immigration Legislation (migr_eil), (2018), Eurostat, https:/ / ec.europa.eu/eu rostat/cache/metadata/en/migr_eil_esms.htm, accessed 23.07.2020.

Foreign population, (2018), Organisation for Economic Co-operation and Development [OECD], https://data.oecd.org/migration/foreign-population.htm\#indicator-chart, accessed 23.07.2020.

Human Development Report 2015. Work for Human Development, (2015). United Nations Development Programme [UNDP]. United Nations Development Programme, New York.

Human Development Reports, (2018), United Nations Development Programme [UNDP], http:/ / hdr.undp.org/en/data\#, accessed 4.07.2020.

International migrant stocks, (2020), IOM's Global Migration Data Analysis Centre [IOM's GMDAC], https://migrationdataportal.org/themes/international-migrant-stocks, accessed 23.07.2020.

International Migration Database. Inflows of foreign population by nationality, (2018), Organisation for Economic Co-operation and Development [OECD.Stat], http://stats. oecd.org/\#, accessed 23.07.2020.

International migration flows to and from selected countries: the 2015 revision, (2015), United Nations Department of Economic and Social Affairs [UN DESA], United Nations, New York, http://www.un.org/en/development/desa/population/migration/data/em pirical2/docs/migflows2015documentation.pdf, , accessed 4.07.2020.

International Migration Outlook 2012, (2012), Organisation for Economic Co-operation and Development [OECD], OECD Publishing, Paris.

List of OECD Member countries - Ratification of the Convention on the OECD, (2018), Organisation for Economic Co-operation and Development [OECD], https://www. oecd.org/about/members-and-partners/, accessed 23.07.2020.

Migration and Home Affairs. Third-country national, (2018), European Commission, Retrieved from https://ec.europa.eu/home-affairs/content/third-country-national_en, accessed 10.11.2019.

Migration Governance Framework, (2019), IOM UN MIGRATION, https://www.iom.int/ sites/default/files/about-iom/migof_brochure_a4_en.pdf, accessed 23.07.2020.

Net migration rate. 1 definition, (2019), United Nations Statistics Division [UNSD], http:/ / data.un.org/Glossary.aspx?q=Net+migration+rate+(per+1\%2C000+population, accessed 4.07.2020.

Permanent immigrant inflows (indicator), (2018), Organisation for Economic Co-operation and Development [OECD]. doi: 10.1787/304546b6-en.

Society at Glance 2016. OECD social indicators, (2016), Organisation for Economic Cooperation and Development [OECD], OECD Publishing, Paris.

Third country nationals found to be illegally present - annual data (rounded), (2020), Eurostat, http:/ / appsso.eurostat.ec.europa.eu/nui/show.do?dataset=migr_eipre\&lang=en, accessed 23.07.2020. 
Third country nationals ordered to leave - annual data (rounded), (2017), Eurostat, http://app sso.eurostat.ec.europa.eu/nui/show.do?dataset=migr_eiord\&lang=en,

Third country nationals refused entry at the external borders - annual data (rounded), (2020), Eurostat, http://appsso.eurostat.ec.europa.eu/nui/show.do?dataset=migr_eirfs\&lang =en, accessed 23.07.2020.

Third countrynationals returned following anorder toleave-annual data (rounded),(2017), Eurostat, http:/ / appsso.eurostat.ec.europa.eu/nui/show.do?dataset=MIGR_EIRTN\&lang $=$ en, accessed 23.07.2020.

Katarzyna Woźniak, Ph.D. of Social Science, an assistant professor in the Department of Macroeconomics and Development Studies (Institute of Economics, the Poznań University of Economics and Business). Research interests: human capital, labour market, migration and its determinants, migration policy. 